University of Nebraska - Lincoln

DigitalCommons@University of Nebraska - Lincoln

2000

\title{
Mirroring and Mimicking of Partial Cross Sections in the Vicinity of a Resonance
}

Chien-Nan Liu

University of Nebraska-Lincoln

Anthony F. Starace

University of Nebraska-Lincoln, astarace1@unl.edu

Follow this and additional works at: https://digitalcommons.unl.edu/physicsstarace

Part of the Physics Commons

Liu, Chien-Nan and Starace, Anthony F., "Mirroring and Mimicking of Partial Cross Sections in the Vicinity of a Resonance" (2000). Anthony F. Starace Publications. 172.

https://digitalcommons.unl.edu/physicsstarace/172

This Article is brought to you for free and open access by the Research Papers in Physics and Astronomy at DigitalCommons@University of Nebraska - Lincoln. It has been accepted for inclusion in Anthony F. Starace Publications by an authorized administrator of DigitalCommons@University of Nebraska - Lincoln. 


\title{
Mirroring and Mimicking of Partial Cross Sections in the Vicinity of a Resonance
}

\author{
Chien-Nan Liu and Anthony F. Starace
}

\begin{abstract}
We review recent analytical work on mirroring and mimicking behavior of resonance profiles in different partial cross sections. This work is related to the work of Fano and Cooper on resonance profiles in total cross sections and that of Starace on resonance profiles in partial cross sections. Applications of the new theories (describing mirroring and mimicking behavior) to recent experimental measurements and theoretical predictions for photoionization, photodetachment, and Auger spectroscopy in the vicinity of resonance structures are discussed.
\end{abstract}

Key words: autoionization, resonance profile, resonance parameter, partial cross section, Auger effect, quantum interference, photoionization, photodetachment, excited atom or ion

\section{INTRODUCTION}

The 1961 paper by Ugo Fano on the behavior of total cross sections in the vicinity of an isolated resonance is probably his most cited work. (1) This work and the more general analysis for multichannel problems carried out with Cooper ${ }^{(2,3)}$ provided experimentalists with three intrinsic parameters with which to characterize isolated resonance profiles in total cross section spectra. These three real parameters are the resonance width $\Gamma$, the profile parameter $q$, and the correlation index $\rho^{2}$. The characteristic asymmetric profile is the signature of the interchannel interference effects involved in the vicinity of a resonance.

At the time Fano and Cooper carried out their analyses, experimentalists primarily measured total cross sections, as in photoabsorption measurements. Analysis of the details of the final state (e.g., as in photoelectron spectroscopy) permitted the measurement of partial cross sections, for which the Fano and Cooper analysis did not apply. Occasionally experimentalists discovered that resonance features either absent from or weak in the total cross sections were prominent in the partial cross sections. Samson and Cairns, ${ }^{(4)}$ for example, remarked upon this in their measurements of the partial cross sections for photoionization of the $5 p$ subshell of $\mathrm{Xe}$, i.e.,

$$
\mathrm{Xe} 5 \mathrm{p}^{6}+\gamma \rightarrow \mathrm{Xe}^{+} 5 \mathrm{p}^{5}\left({ }^{2} \mathrm{P}_{J}\right)+e^{-},
$$

where $J=3 / 2$ or $1 / 2$. They noted that "...at the $543 \AA$ resonance the $\sigma_{3 / 2}$ cross section decreased while the $\sigma_{1 / 2}$ cross section increased by almost the same magnitude. The net result was that the two large resonances [i.e., in each partial cross section] practically annulled each other such that only a weak resonance could be observed in the total cross section curve."

Similarly, Krause et al. $^{(5)}$ found that the absorption spectrum of lead showed almost no evidence of the resonance series, $6 s^{2} 6 p^{2}+\gamma \rightarrow 6 s 6 p^{2}\left({ }^{4} P_{1 / 2}\right) n p_{3 / 2}$, whereas this series is prominent in the $J=3 / 2,1 / 2$ partial cross sections:

$$
6 s^{2} 6 p^{2}+\gamma \rightarrow 6 s^{2} 6 p\left({ }^{2} P_{J}\right)+e^{-}
$$

The explanation for this is that the series appears as resonance peaks in the ${ }^{2} \mathrm{P}_{3 / 2}$ partial cross section but as resonance windows in the ${ }^{2} \mathrm{P}_{1 / 2}$ partial cross section, almost canceling in the total cross section.

In recent years, as experimental energy resolutions and detection capabilities have improved, the instances in which such mirroring behavior of resonance features in different partial cross sections occur have increased, not only in photoionization ${ }^{(6,7)}$ but also in photodetachment of negative ions. ${ }^{(8)}$ Even in Auger spectroscopy, experimentalists have recently found that the typical Lorentzian line profile seen in total cross sections is not appropriate in partial cross sections, where the measurements show asymmetric line profiles, with the differences effectively compensating each other in the summed cross section. ${ }^{(9)}$

The increasing occurrence of such mirroring behavior as experimentalists examine partial cross sections in the neighborhood of resonances can be explained analytically. First, one has to use a theory for resonance line shapes appropriate for partial cross sections. Starace ${ }^{(10)}$ has extended the Fano and Cooper analysis to treat partial cross sections. Second, one has to examine the limiting case in which the Fano and Cooper correlation index, $\rho^{2}$, approaches zero, i.e., the case in which the maximum fractional depth of the minimum of the total cross section in the vicinity of a resonance is small. In this instance mirroring behavior can be shown analytically to occur. ${ }^{(11)}$ The opposite extreme, in which the resonance creates a deep window in the total cross 
section, can be shown theoretically ${ }^{(12)}$ to result in mimicking behavior, i.e., each partial cross section has a similarly shaped window-type line profile.

In what follows we first discuss briefly the theory for resonance profiles appropriate for partial cross sections. ${ }^{(10)}$ We then examine the partial cross section profile formulas for the two limiting cases of $\rho^{2} \rightarrow 0^{(11)}$ and $\rho^{2} \rightarrow 1 .^{(12)}$ Finally, we discuss the implications of the formulas obtained and present some examples.

\section{RESONANCE LINE PROFILES IN PARTIAL CROSS SECTIONS}

Generalization of the Fano and Cooper analyses ${ }^{(1-3)}$ to describe partial cross sections in the vicinity of an isolated resonance was carried out by Starace. ${ }^{(10)}$ Since total cross sections can always be expressed as an incoherent sum of the absolute squares of transition matrix elements from the initial state to scattering eigenstates satisfying standing wave boundary conditions, resonance profiles for total cross sections can be characterized, as in the Fano and Cooper analyses, ${ }^{(1-3)}$ using real parameters. Resonance profiles for partial cross sections require complex parameters, however, owing to the asymptotic boundary conditions that define the partial cross sections.

More specifically, partial cross sections must be obtained as the absolute square of a linear combination of transition matrix elements from the initial state to scattering eigenstates. This linear combination is determined by requiring the final state to satisfy the usual outgoing- or incomingwave scattering boundary conditions. Note that partial cross sections are in general characterized by experimentally measurable quantities corresponding to final states that are not scattering eigenstates. For example, in a photoionization process the experimental measurement might specify the state of the ionic core and the energy of the photoelectron. However, the scattering eigenstates for a photoionization process are in general not characterized by fixed values for these experimentally measurable quantities (i.e., the ionic core state and the photoelectron energy). Consequently, in general the transition matrix element characterized by such fixed asymptotic boundary conditions is a linear combination of transition matrix elements to scattering eigenstates. The linear combination involves in general complex coefficients owing to the incoming- or outgoing-wave boundary conditions that a particular photoionization or scattering process respectively must satisfy.

Starace showed ${ }^{(10)}$ for the case of photoionization of an initial state $\psi_{0}$ that the electric dipole transition amplitude to a particular final state channel $\mu$ having system energy $E$ can be described in the vicinity of a resonance by introducing a single complex parameter $\alpha(\mu E)$, in addition to the Fano profile parameter $q$ :

$$
\left\langle\psi_{0}|\vec{r}| \mu E-\right\rangle=\left\langle\psi_{0}|\vec{r}| \mu E-\right\rangle_{0}\left[1+\frac{\alpha(\mu E)(q+i)}{(\varepsilon-i)}\right]
$$

On the right-hand side of (3) the subscript zero on the transition matrix element indicates that it is the transition matrix element outside the energy region of the resonance, and the minus sign inside the ket indicates that the final state $\mu E$ satisfies incoming-wave boundary conditions. The reduced energy, $\varepsilon$, is defined by

$$
\varepsilon \equiv \frac{E-E_{r s}(E)}{(1 / 2) \Gamma(E)}
$$

where the width $\Gamma(E)$ and the resonance energy $E_{\text {res }}(E)$ do in principle depend on the system energy $E$. However, if the resonance is narrow, this energy dependence is negligible and may be ignored. A similar statement may be made regarding the energy dependence of the parameter $\alpha(\mu E)$ in (3). Although for consistency of notation with Ref. 10 we shall retain specification of $E$ in $\alpha(\mu E)$, readers may assume that for narrow resonances $E$ is evaluated at the resonance energy.

With the transition matrix element in (3) one can describe the behavior of any measurable photoionization or photodetachment quantity in the vicinity of an isolated resonance. Starace ${ }^{(10)}$ analyzed the behavior of partial cross sections and partial cross section branching ratios in the vicinity of an isolated resonance. Kabachnik and Sazhina ${ }^{(13)}$ analyzed the behavior of photoelectron angular distributions and spin polarizations in the vicinity of an isolated resonance. (The connection between the formulations of Refs. 10 and 13 is discussed in Sec. VI.C of Ref. 10.)

A key difference between the profile parameter $q$, the resonance energy $E_{\text {res }}$, and the width parameter $\Gamma$ on the one hand, and the new parameter $\alpha(\mu E)$ on the other hand, is that the latter depends on the particular scattering channel $\mu$. Since $\alpha(\mu E)$ is complex, for a given resonance there are thus twice as many parameters as there are scattering channels. As shown in Ref. 10, the parameters $\alpha(\mu E)$ do satisfy certain sum rules, which reduces the number of independent parameters somewhat. Nevertheless, determination of all parameters $\alpha(\mu E)$ for a particular resonance amounts to a solution of the complete scattering problem in the vicinity of the resonance.

The partial cross section for the channel $\mu$ with system energy $E$ is proportional to the absolute square of the amplitude in (3), i.e.,

$$
\begin{aligned}
& \mid\left\langle\psi_{0}|\vec{r}| \mu E-\right\rangle^{2} \\
& =\frac{\left|\left\langle\psi_{0}|\vec{r}| \mu E-\right\rangle_{0}\right|^{2}}{1+\varepsilon^{2}} \times\left\{\varepsilon^{2}+2 \varepsilon[q \operatorname{Re} \alpha(\mu E)-\operatorname{Im} \alpha(\mu E)]\right. \\
& \left.+\left[1-2 q \operatorname{Im} \alpha(\mu E)-2 \operatorname{Re} \alpha(\mu E)+\left(q^{2}+1\right)|\alpha(\mu E)|^{2}\right]\right\} .
\end{aligned}
$$

One sees in (5) that this partial cross section varies with the reduced energy $\varepsilon$ across the resonance in the rather ubiquitous way that essentially all physical quantities in the vicinity of a resonance do, i.e., in the denominator there is a 
$1+\varepsilon^{2}$ dependence, and in the numerator there is a quadratic polynomial in $\varepsilon$. A measurement of the partial cross section corresponding to the transition probability in (5) in the vicinity of the resonance would determine $\alpha(\mu E)$ completely (assuming one knows the profile parameter $q$, the resonance energy $E_{\text {res }}$ and the width $\Gamma$ ). In the limit that there exists only a single channel, the parameter $\alpha(\mu E)$ becomes equal to unity and (5) reduces to the form of the Fano line profile,

$$
\left.\left|\left\langle\psi_{0}|\vec{r}| \mu E-\right\rangle^{2}=\right|\left\langle\psi_{0}|\vec{r}| \mu E-\right\rangle_{0}\right|^{2} \frac{(q+\varepsilon)^{2}}{1+\varepsilon^{2}} .
$$

Note that (6) becomes zero when $\varepsilon=-q$.

In order to provide a physical interpretation of the channel parameter $\alpha(\mu E)$, we must first discuss the multichannel generalization of the Fano profile result in (6), which involves a new parameter, the correlation index $\rho^{2}:^{(3)}$

$$
\sigma_{T O T}=\sigma_{T O T}^{0}\left[\rho^{2} \frac{(q+\varepsilon)^{2}}{1+\varepsilon^{2}}+1-\rho^{2}\right]
$$

In (7), $\sigma_{\text {TOT }}{ }^{0}$ is the total cross section in the absence of the resonance and $\rho^{2}$ is the maximum fractional depth of the minimum of the total cross section in the vicinity of a resonance. ${ }^{(3)}$ Note that $\rho^{2}$ takes the values $0 \leq \rho^{2} \leq 1$. The Fano treatment ${ }^{(1)}$ for the interaction between the resonance and $N$ continuum channels consists of transforming the $N$ degenerate continuum states at a given total energy $E$ to $N$ new orthonormal states having only a single member, labeled $\lambda=1$, which interacts with the resonance. In (7), the contribution from the $\lambda=1$ channel is similar to the original Fano profile formula in (6), while the other channels (i.e., $2 \leq \lambda \leq N$ ) are unaffected by the resonance and produce a constant background. One observes that for $\varepsilon=-q$, the $\lambda=1$ channel's cross section will go to zero. The parameter $\rho^{2}$ gives the fractional depth of this minimum relative to the value of the total cross section outside the resonance energy region. One may interpret the channel parameter $\alpha(\mu E)$ in (3) as the fraction of the transition amplitude from the initial state $\psi_{0}$ to the final state $\mu E$, which passes through the channel $\lambda=1$, which is the only channel (in the $\lambda$ basis set) that interacts with the resonance.

Typically an experimentally measured partial cross section is given by a sum of the channel cross sections, which are proportional to the absolute square transition amplitudes in (5). (For example, in a photoionization measurement of the partial cross section for producing a particular state of the ion, one must sum the channel partial cross sections corresponding to (5) over all $\mu$ involving that state of the ion; the channels will still have differing angular momenta for the photoelectron and for its coupling to the ion.) If we label the subset of channels that contribute to this sum as $P$, then the partial cross section $P$ is given by

$$
\begin{aligned}
& \sigma_{P}=\frac{\sigma_{P}^{0}}{1+\varepsilon^{2}}\left\{\varepsilon^{2}+2 \varepsilon\left(q \operatorname{Re}\langle\alpha\rangle_{P}-\operatorname{Im}\langle\alpha\rangle_{P}\right)\right. \\
& \left.+\left[1-2 q \operatorname{Im}\langle\alpha\rangle_{P}-2 \operatorname{Re}\langle\alpha\rangle_{P}+\left(q^{2}+1\right)\left\langle|\alpha|^{2}\right\rangle_{P}\right]\right\},
\end{aligned}
$$

where the averages of the $\alpha(\mu E)$ parameters appearing in (8) are defined as follows:

$$
\langle\alpha\rangle_{P} \equiv \frac{\sum_{\mu \in P} \alpha(\mu E)\left|\left\langle\psi_{0}|\vec{r}| \mu E-\right\rangle_{0}\right|^{2}}{\sum_{\mu \in P}\left|\left\langle\psi_{0}|\vec{r}| \mu E-\right\rangle_{0}\right|^{2}}
$$

and

$$
\left\langle|\alpha|^{2}\right\rangle_{P} \equiv \frac{\sum_{\mu \in P}|\alpha(\mu E)|^{2}\left|\left\langle\psi_{0}|\vec{r}| \mu E-\right\rangle_{0}\right|^{2}}{\sum_{\mu \in P}\left|\left\langle\psi_{0}|\vec{r}| \mu E-\right\rangle_{0}\right|^{2}}
$$

Because in (8) there are now three unknowns $\left(\operatorname{Re}\langle\alpha\rangle_{P^{\prime}}\right.$ $\operatorname{Im}\langle\alpha\rangle_{p}$, and $\left\langle|\alpha|^{2}\right\rangle_{p}$ ) in addition to the usual parameters $q$, $E_{\text {res }}$ and $\Gamma$, a measurement of this partial cross section does not determine all three, but only certain linear combinations of them. Specifically, we may rewrite (8) as

$$
\sigma_{P}=\frac{\sigma_{P}^{0}}{1+\varepsilon^{2}}\left\{\varepsilon^{2}+2 \varepsilon C_{1}+\left(1+C_{2}\right)\right\}
$$

where $C_{1}$ and $C_{2}$ are new parameters that can be determined by a measurement of $\sigma_{P}$ in the vicinity of the resonance. Their expressions in terms of $\langle\alpha\rangle_{P}$ and $\left\langle|\alpha|^{2}\right\rangle_{P}$ may be obtained by direct comparison of (8) and (11). Note that although (7) can be reduced to the same parameterized expression as (11), the parameters have very different physical interpretations. That is why although one can use the multichannel generalization of the Fano profile formula in (7) to fit resonance profiles in partial cross sections, the parameters $q$ and $\rho^{2}$ thus obtained are meaningless, i.e., they are not the parameters $q$ and $\rho^{2}$ one obtains by fitting (7) to the resonance profile in the total cross section.

\section{MIRRORING OF RESONANCE PROFILES IN PAR- TIAL CROSS SECTIONS}

We assume that all the channels contributing to a given total cross section are divided into two groups, which we label $P$ and $Q$. That is,

$$
\sigma_{T O T}=\sigma_{P}+\sigma_{Q}
$$

where $\sigma_{P}$ behaves in the vicinity of the resonance according to (8). $\sigma_{Q}$ has an identical form to that in (8), but with $P$ replaced by $Q$. Note that the division of the total cross section 
into a group $P$ and a group $Q$ is arbitrary; our results below apply for any such division. Before proceeding with the proof of the mirroring of $\sigma_{P}$ and $\sigma_{Q}$, we note that the averages of the $\alpha(\mu E)$ parameters within the groups of channels labeled $P$ and $Q$ are related to one another by means of the correlation index $\rho^{2}$, as shown in Ref. 10:

$$
\begin{gathered}
\sigma_{P}^{0}\left\langle|\alpha|^{2}\right\rangle_{P}+\sigma_{Q}^{0}\left\langle|\alpha|^{2}\right\rangle_{Q}=\rho^{2} \sigma_{T O T}^{0} \\
\sigma_{P}^{0}\langle\alpha\rangle_{P}+\sigma_{Q}^{0}\langle\alpha\rangle_{Q}=\rho^{2} \sigma_{T O T}^{0} .
\end{gathered}
$$

The mirroring behavior of $\sigma_{P}$ and $\sigma_{Q}$ occurs empirically for resonances having a correlation index $\rho^{2}$ close to zero. However, taking this limit in (13) and (14) appears to give the trivial result that all of the $\alpha$ parameters and their averages may be set to zero. This simple analysis is incorrect because it fails to take into account the behavior of the profile parameter $q$ as $\rho^{2} \rightarrow 0$.

As shown in Ref. $11, q \rho^{2} \rightarrow 0$ in the limit when $\rho^{2} \rightarrow 0$, but $q^{2} \rho^{2}$ remains finite. In fact, as implied by (7), $q^{2} \rho^{2}$ measures the fractional rise of the cross section in the vicinity of a resonance above what would be the case in the absence of the resonance. ${ }^{(3)}$ Taking the behaviors of $q \rho^{2}$ and $q^{2} \rho^{2}$ into account, (13) and (14) imply that as $\rho^{2} \rightarrow 0$, we have

$$
\begin{gathered}
\lim _{\rho \rightarrow 0} \sigma_{Q}^{0} q^{2}\left\langle|\alpha|^{2}\right\rangle_{Q}=\rho^{2} q^{2} \sigma_{T O T}^{0}-\sigma_{P}^{0} q^{2}\left\langle|\alpha|^{2}\right\rangle_{P}, \\
\lim _{\rho \rightarrow 0} q \sigma_{Q}^{0}\langle\alpha\rangle_{Q}=-q \sigma_{P}^{0}\langle\alpha\rangle_{P} .
\end{gathered}
$$

In the limit when $\rho^{2} \rightarrow 0$, the partial cross sections can now be written as

$$
\begin{gathered}
\lim _{\rho \rightarrow 0} \sigma_{P}=\sigma_{P}^{0}+\frac{\sigma_{P}^{0}}{1+\varepsilon^{2}}\left\{2 q\left[\varepsilon \operatorname{Re}\langle\alpha\rangle_{P}-\operatorname{Im}\langle\alpha\rangle_{P}\right]+q^{2}\left\langle|\alpha|^{2}\right\rangle_{P}\right\}, \\
\lim _{\rho \rightarrow 0} \sigma_{Q}=\sigma_{Q}^{0}+\frac{\sigma_{P}^{0}}{1+\varepsilon^{2}}\left\{-2 q\left[\varepsilon \operatorname{Re}\langle\alpha\rangle_{P}-\operatorname{Im}\langle\alpha\rangle_{P}\right]\right. \\
\left.-q^{2}\left\langle|\alpha|^{2}\right\rangle_{P}+\rho^{2} q^{2}\left(\sigma_{T O T} / \sigma_{P}^{0}\right)\right\} .
\end{gathered}
$$

Summing (17) and (18), we find

$$
\lim _{\rho \rightarrow 0} \sigma_{T O T}=\lim _{\rho \rightarrow 0}\left(\sigma_{P}+\sigma_{Q}\right)=\sigma_{T O T}^{0}\left(1+\frac{\rho^{2} q^{2}}{1+\varepsilon^{2}}\right) .
$$

One sees that, unlike (7), (19) does not have an interference term proportional to $\varepsilon /\left(1+\varepsilon^{2}\right)$. The term proportional to $\rho^{2} q^{2}$ represents a symmetric Lorentzian autoionization profile. Nevertheless, although there are no interference effects in the total cross section, (17) and (18) generally represent asymmetric line profiles, which indicate interference behavior in the partial cross sections. Also, while (19) implies that the total cross section cannot be zero, (17) and (18) allow the possibility that the partial cross sections may have a zero minimum. More specifically, the energy-dependent parts of $\sigma_{P}$ in (17) and $\sigma_{Q}$ in (18) are equal in magnitude and opposite in sign except for the term $\rho^{2} q^{2} \sigma_{\text {TOT }} /\left(1+\varepsilon^{2}\right)$ in (18). Thus, one sees analytically that the two cross sections mirror each other's behavior. Also, since the division of the total cross section into the two groups $P$ and $Q$ is arbitrary, mirroring behavior between any two such groups of individual cross sections is implied. This mirroring will be more pronounced the smaller the term involving $\rho^{2} q^{2}$ in (18) is, or, alternatively, the smaller the effect of the resonance on the total cross section is.

\section{MIMICKING OF RESONANCE PROFILES IN PAR- TIAL CROSS SECTIONS}

In the opposite limit that $\rho^{2} \rightarrow 1$, the resonance cuts a deep window-type profile in the total cross section, which for $\rho^{2}=1$ becomes zero. As shown in Ref. 12, the easiest way to analyze the partial cross sections in this limit is to consider the form for $\sigma_{P}$ in (11). According to Ref. 10, $\sigma_{Q}$ may then be written in the form

$$
\begin{aligned}
\sigma_{Q}=\frac{\sigma_{Q}^{0}}{1+\varepsilon^{2}}\left\{\varepsilon^{2}+2 \varepsilon\left[q \rho^{2}(1+r)-r C_{1}\right]\right. \\
\left.+\left[1+\left(q^{2}-1\right) \rho^{2}(1+r)-r C_{2}\right]\right\}
\end{aligned}
$$

where

$$
r \equiv\left(\frac{\sigma_{P}^{0}}{\sigma_{Q}^{0}}\right)
$$

Now $C_{1}$ and $C_{2}$ can be further parameterized as in Eqn. (52) of Ref. 10:

$$
C_{1} \equiv \frac{R}{(1+r)^{1 / 2}} \sin \theta \sin \phi+q \rho^{2},
$$

$$
C_{2} \equiv-1+R^{2} \sin ^{2} \theta \cos ^{2} \phi+C_{1}^{2},
$$

where $\theta$ and $\phi$ are parametric variables and 


$$
R^{2} \equiv\left(1+\frac{1}{r}\right)\left[1+\left(q^{2}-1\right) \rho^{2}-q^{2} \rho^{4}\right]
$$

From (24), $R \rightarrow 0$ as $\rho^{2} \rightarrow 1$. From (22) and (23), one then has $C_{1}=q$ and $C_{2}=q^{2}-1$. Then

$$
\frac{\sigma_{P}}{\sigma_{P}^{0}}=\frac{\sigma_{Q}}{\sigma_{Q}^{0}}=\frac{(\varepsilon+q)^{2}}{1+\varepsilon^{2}} .
$$

Since the division of the total cross section into groups $P$ and $Q$ is arbitrary, one sees that each partial cross section has the same energy dependence in the neighborhood of a resonance for which $\rho^{2} \rightarrow 1$. That is, the partial cross sections (and the total cross section) mimic each other.

\section{EXAMPLES}

We illustrate mirroring behavior with examples from our recent comprehensive eigenchannel R-matrix study of photodetachment of $\mathrm{He}^{-}\left(1 \mathrm{~s} 2 \mathrm{~s} 2 \mathrm{p}^{4} \mathrm{P}^{0}\right){ }^{(12)}$ First, though, we must discuss the various kinds of partial cross sections that one may define for this system. Since the ground state of $\mathrm{He}^{-}$ is a ${ }^{4} \mathrm{P}^{0}$ state, electric dipole selection rules and $L S$ coupling permit only ${ }^{4} L^{e}$ final states, where $L=0,1$, and 2 . The cross section $\sigma\left({ }^{4} L^{e}\right)$ for the ${ }^{4} L^{e}$ final state corresponds to the following process:

$$
\mathrm{He}^{-}\left(1 \mathrm{~s} 2 \mathrm{~s} 2 \mathrm{p}^{4} \mathrm{P}^{0}\right)+\gamma \rightarrow \sum_{n l}^{n l(\max )} \sum_{l^{\prime}} \mathrm{He}\left(\operatorname{ls} n l^{3} l\right) \varepsilon(n l) l^{\prime}\left({ }^{4} L^{e}\right)
$$

where $\varepsilon(n l)$ is the kinetic energy of the detached electron when the He atom is in the state $\mathrm{He}\left(1 \mathrm{~s} n l{ }^{3} l\right) ; n l$ takes the values $2 s \leq n l \leq 5 f$ in the energy region with which Ref. 12 is concerned and, for a given photon energy, $n l(\max )$ is the highest of these states that is allowed by energy conservation. Also, for a given pair of values $(l, L)$, the detached electron's orbital angular momentum $l^{\prime}$ takes all values allowed by parity and orbital angular momentum conservation.

The quartet doubly excited states of $\mathrm{He}^{-}$populated in photodetachment processes have well-defined term values ${ }^{4} L^{e}$. It is theoretically useful to examine their effects on partial cross sections having not only a well-defined term level, but also a well-defined state of the $\mathrm{He}$ atom. We thus define $\sigma\left(n l,{ }^{4} L^{e}\right)$ to correspond to the following process:

$$
\mathrm{He}^{-}\left(\operatorname{ls} 2 \mathrm{~s} 2 \mathrm{p}^{4} \mathrm{P}^{0}\right)+\gamma \rightarrow \sum_{l^{\prime}} \mathrm{He}\left(\mathrm{ls} n l^{3} l\right) \varepsilon(n l) l^{\prime}\left({ }^{4} L^{e}\right)
$$

In Fig. 1, we compare the total cross section for ${ }^{4} S^{e}$ final states $\sigma\left({ }^{4} S^{e}\right)$ and two partial cross sections,

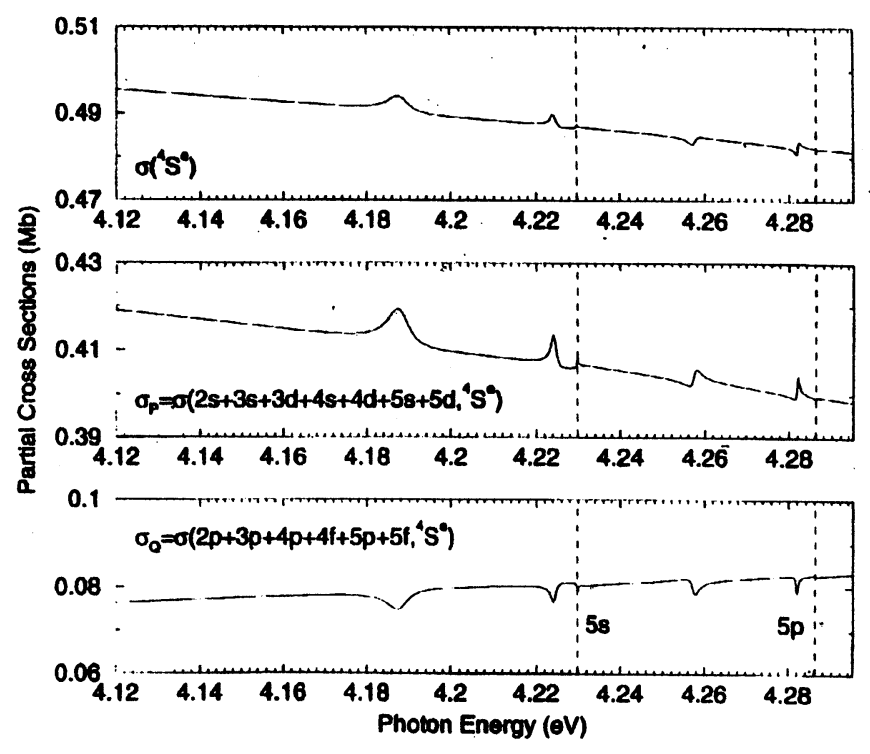

Figure 1. Mirroring behavior in the ${ }^{4} S^{e}$ partial cross sections in the region of the He $(n=5)$ thresholds. (a) $\sigma\left({ }^{4} S^{e}\right)$. (b) $\sigma_{P}=\Sigma_{n l} \sigma\left(n l,{ }^{4} S^{e}\right)$ for $2 \mathrm{~s} \leq n l \leq 5 \mathrm{~d}$ and $l=$ even. (c) $\sigma_{Q}=$ $\Sigma_{n l} \sigma\left(n l,{ }^{4} S^{e}\right)$ for $2 \mathrm{p} \leq n l \leq 5 \mathrm{f}$ and $l=$ odd. The vertical dashed lines indicate the locations of the $\mathrm{He}\left(1 \mathrm{snl} l^{3} L\right)$ thresholds for $n l=5 \mathrm{~s}$ and $5 \mathrm{p}$. Note that $\left.\sigma^{4} \mathrm{~s}^{e}\right)=\sigma_{P}+\sigma_{Q}$ and that $\sigma_{P}$ and $\sigma_{Q}$ mirror one another relative to changes in $\left.\sigma^{4} S^{e}\right)$ in the vicinity of the resonances, as predicted analytically in Ref. 11.

$$
\sigma_{P}=\sum_{n l}^{l=\text { even }} \sigma\left(n l,{ }^{4} \mathrm{~S}^{e}\right)
$$

and

$$
\sigma_{Q}=\sum_{n l}^{l=\text { odd }} \sigma\left(n l,{ }^{4} S^{e}\right)
$$

where, of course, $\sigma\left({ }^{4} S^{e}\right)=\sigma_{P}+\sigma_{Q}$.

One sees from Fig. la that $\sigma\left({ }^{4} S^{e}\right)$ shows minimal evidence of resonance structure, indicating that $\rho \approx 0$, so that the predictions of Ref. 11 are applicable to this case. Thus, when we split $\sigma\left({ }^{4} S^{e}\right)$ into two groups, as shown in Fig. l, we see that $\sigma_{P}$ and $\sigma_{Q}$ mirror each other in the vicinity of the ${ }^{4} S^{e}$ doubly excited resonances relative to the changes in $\sigma_{\text {Total }}$ (i.e., $\sigma\left({ }^{4} S^{e}\right)$ ) in the vicinity of the resonances. If the effects of the resonances on $\sigma\left({ }^{4} S^{e}\right)$ were nil, then the mirroring of $\sigma_{P}$ and $\sigma_{Q}$ would be exact. ${ }^{(11)}$ Note that the three panels in Fig. $\mathrm{l}$ are plotted on the same scale in order to facilitate comparisons of changes in the vicinities of the resonances. Remember also that the division of $\sigma_{\text {Total }}$ into two groups, $P$ and $Q$, 


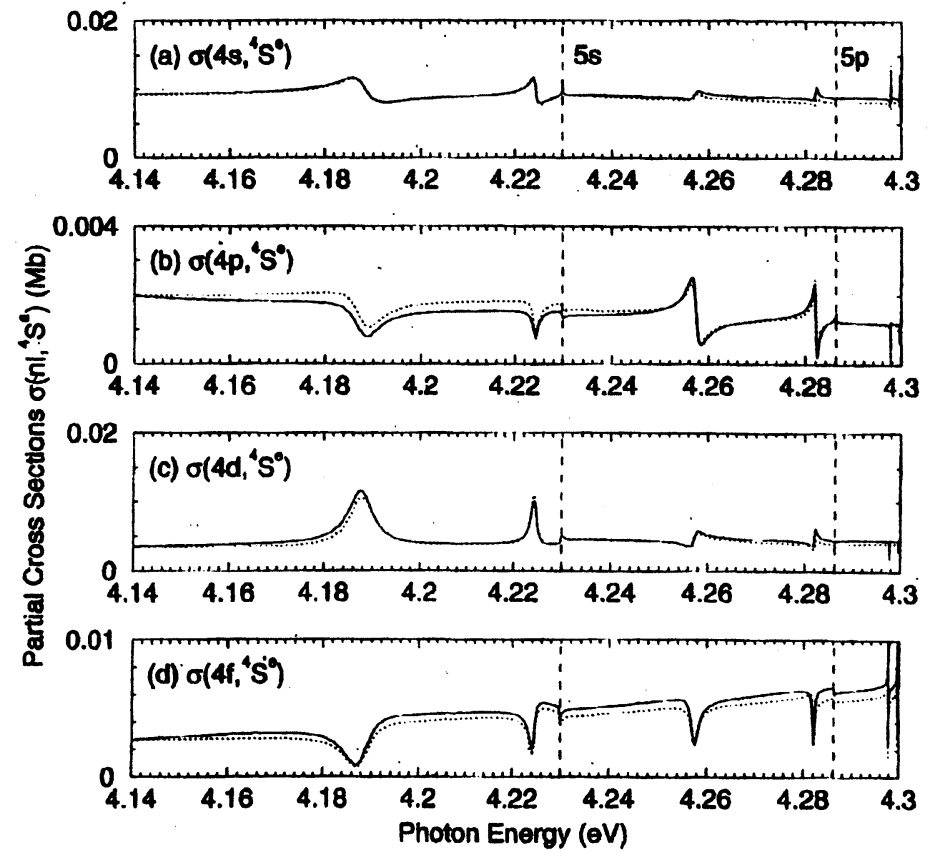

Figure 2. $\sigma\left(n l,{ }^{4} S^{e}\right)$ partial cross sections in the vicinity of the He $(n=5)$ thresholds. (a) $\sigma\left(4 \mathrm{~s},{ }^{4} S^{e}\right)$. (b) $\sigma\left(4 \mathrm{p},{ }^{4} S^{e}\right)$. (c) $\sigma(4 \mathrm{~d}$, $\left.{ }^{4} S^{e}\right)$. (d) $\sigma\left(4 \mathrm{f},{ }^{4} S^{e}\right)$. The vertical dashed lines indicate the locations of the He $\left(l s n l^{3} L\right)$ thresholds for $n l=5 \mathrm{~s}$ and $5 \mathrm{p}$.

is quite arbitrary: results similar to those shown in Fig. 1 may be found for other choices for $P$ and $Q$. Therefore, mirroring behavior between individual partial cross sections is implied, as illustrated in Fig. 2 . We see that the $\sigma\left(4 \mathrm{p},{ }^{4} \mathrm{~S}^{e}\right)$ and $\sigma\left(4 \mathrm{~d},{ }^{4} S^{e}\right)$ partial cross sections, for example, are nearly mirror images of each other in the vicinity of the resonances. While these resonances are not prominent in the cross section $\sigma\left({ }^{4} S^{e}\right)$ (cf. Fig. la), the $\sigma\left(4 l,{ }^{4} S^{e}\right)$ partial cross sections shown in Fig. 2 are dominated by the doubly excited resonances, exhibiting such interference effects as asymmetric peaks and, in some cases, nearly zero minima.

Mimicking behavior occurs whenever a resonance causes a deep window in the total cross section. Such an occurrence is expected to be unusual if the resonance is highly excited, since then its interaction with continuum channels having low levels of excitation of the atom is normally expected to be small. However, Ref. 12 predicted three notable exceptions in the $\sigma\left({ }^{4} \mathrm{P}^{e}\right)$ cross section in the vicinity of the $4 \mathrm{~s}$ and $4 \mathrm{p}$ thresholds, as shown in Fig. 3a. These exceptional doubly excited resonances are located at 3.81,3.945, and $3.965 \mathrm{eV}$, and have nearly zero minima, indicating a correlation index $\rho^{2}$ close to unity. ${ }^{(2,3)}$ As discussed in Section 4, for a resonance having $\rho^{2} \rightarrow 1$, variations of different partial cross sections in the vicinity of the resonance are in phase. That is, they mimic one another in the neighborhood of the resonance. The $\sigma\left(n l,{ }^{4} \mathrm{P}^{e}\right)$ partial cross sections in the neighbor-
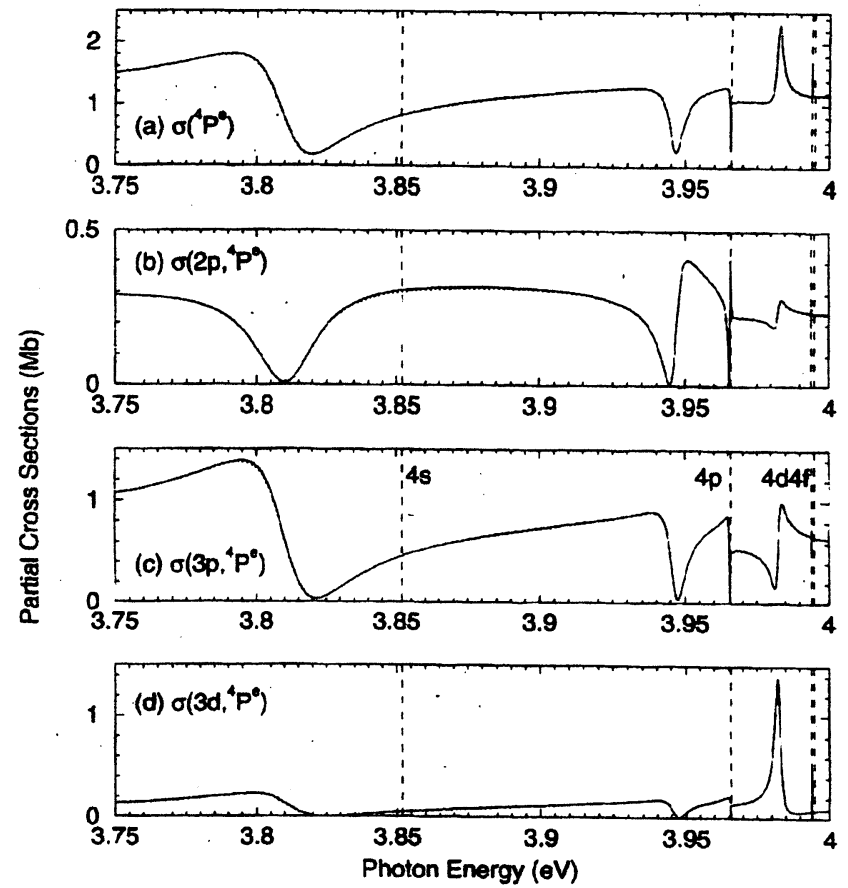

Figure 3. $\sigma\left({ }^{4} \mathrm{P}^{e}\right)$ and $\sigma\left(n l,{ }^{4} \mathrm{P}^{e}\right)$ partial cross sections in the vicinity of the He $(n=4)$ thresholds. (a) $\sigma\left({ }^{4} \mathrm{P}^{e}\right)$. (b) $\sigma(2 \mathrm{p}$, $\left.{ }^{4} \mathrm{P}^{e}\right)$. (c) $\sigma\left(3 \mathrm{p},{ }^{4} \mathrm{P}^{e}\right)$. (d) $\sigma\left(3 \mathrm{~d},{ }^{4} \mathrm{P}^{e}\right)$. The vertical dashed lines indicate the locations of the $\mathrm{He}\left(1 \mathrm{~s} n l^{3} \mathrm{~L}\right)$ thresholds for $4 \mathrm{~s} \leq$ $n l \leq 4 \mathrm{f}$. Note the mimicking behavior of the resonances located at $\hbar \omega=3.81 \mathrm{eV}, 3.945 \mathrm{eV}$, and $3.965 \mathrm{eV}$.

hood of these three resonances are shown in Fig. 3 to illustrate such mimicking behaviors.

\section{DISCUSSION AND CONCLUSIONS}

Mirroring behavior among partial cross sections in the vicinity of a resonance is a common occurrence in the highenergy part of spectra, where the resonance state usually has a correlation index $\rho^{2}$ close to zero due to its weak interaction with the dominant ionization (or detachment) continuum channels associated with the lowest energy states of the residual ion (or atom). However, mirroring behavior in the partial cross sections becomes less obvious if the resonance profiles in the total cross sections are dominated by the symmetric component $\rho^{2} q^{2} /\left(1+\varepsilon^{2}\right.$ ) (cf. (19)). Nevertheless, resonances in different partial cross sections are expected to show different asymmetries. One can find an example in a recent experimental measurement of $\mathrm{Li}$ photoionization, where the resonance profiles of the $2 s^{2} 2 p$ triply excited state in the single and double ionization partial cross sections exhibit opposite asymmetries. ${ }^{(14)}$

The analytic proof of Ref. 11 provides a theoretical underpinning to experimental findings that sometimes resonance states that do not appear in total cross sections do appear in partial cross sections. One finds a most recent example in a 
high-resolution measurement of the low-energy photoionization spectrum of $\mathrm{Ar}^{\left({ }^{(15)}\right.}$ in which two resonances in the $3 p_{3 / 2,1 / 2}^{-1}$ partial cross sections exhibit mirroring profiles, resulting in complete cancellation in the total photoionization cross section. The near-zero correlationindexes of the resonance states are due to their $L S$-forbidden character.

An important implication of mirroring behavior is that the intrinsic interference effects due to two indistinguishable quantum paths involved in a resonance are not negligible, even though the symmetric resonance profiles suggest otherwise. For example, in studies of resonant Auger spectra, where prominent symmetric resonance profiles in the total cross section dominate, a two-step sequential model has been commonly used to carry out calculations. However, recent theoretical and experimental measurements show interference effects in the partial cross sections ${ }^{(9)}$ as well as mirroring behaviors among different partial cross sections. ${ }^{(16)}$ Therefore, using a two-step sequential model to describe the autoionization resonances in photofragmentation processes does not give a correct picture, even if the resonance profile in the total cross section is Lorentzian.
We conclude by noting that this recent work on mirroring ${ }^{(11)}$ and mimicking ${ }^{(12)}$ shows that the subject of resonance line profiles begun long ago by Fano and Cooper ${ }^{(1-3)}$ is still a fruitful one for analytic theoretical work and that it continues to provide useful tools for experimental measurements. Specifically, measurements of or predictions for partial cross sections provide information on quantum interference effects that may be absent from the total cross section. Also, predicted mirroring and mimicking behaviors provide a self-consistency test for experimental measurements of different partial cross sections, especially for relative measurements.

\section{Acknowledgments}

CNL thanks T.W. Gorczyca for valuable discussions concerning Auger spectra. This work has been supported in part by the Division of Chemical Sciences, Office of Basic Energy Sciences, Office of Science, U.S. Department of Energy.

Received 4 August 2000.

\section{Résumé \\ Les profils de résonance des sections efficaces ont parfois une allure identique (ce comportement est alors dit "mimant") ou sont parfois images l'une de l'autre comme dans un miroir (comportement dit "miroitant"). Nous passons en revue des travaux analytiques récents sur les comportements "miroitant" et "mimant" des profils de résonance dans les différentes sections efficaces partielles. Ce travail est basé sur ceux de Fano et Cooper sur les profils de résonance dans les sections efficaces totales et celui de Starace sur les profils de résonance dans les sections efficaces partielles. Nous analysons les applications des nouvelles théories (qui décrivent ces comportements "miroitant" et "mimant") aux récentes mesures expérimentales et prédictions théoriques sur la photoionisation, le "photo-détachement" et la spectroscopie Auger au voisinage des structures de résonance.}

\section{References}

l. U. Fano, Phys. Rev. 124, 1866 (1961).

2. U. Fano and J.W. Cooper, Phys. Rev. A 137, 1364 (1965).

3. Idem, Rev. Mod. Phys. 40, 441 (1968).

4. J.A.R. Samson and R.B. Cairns, Phys. Rev. 173, 80 (1968).

5. M.O. Krause, F. Cerrina, A. Fahlman, and T.A. Carlson, Phys. Rev. Lett. 51, 2093 (1983).

6. A. Menzel, S.P. Frigo, S.B. Whitfield, C.D. Caldwell, and M.O. Krause, Phys. Rev. A 54, 2080 (1996).

7. S. Benzaid et al., Phys. Rev. A 54, R2537 (1996).

8. G. Haeffler, I.Yu. Kiyan, D. Hanstorp, and D.J. Pegg, Phys. Rev. A 57, 2216 (1998).

9. R. Camilloni et al., Phys. Rev. Lett. 77, 2646 (1996).

10. A.F. Starace, Phys. Rev. A 16, 231 (1977); Bull. Am. Phys. Soc. 19, 1203 (1974).

11. C.-N. Liu and A.F. Starace, Phys. Rev. A 59, R1731 (1999).

12. Ibid. 60, 4647 (1999).

13. N.M. Kabachnik and I.P. Sazhina, J. Phys. B 9, 1681 (1976). 
14. R. Wehlitz, M.T. Huang, K.A. Berrington, S. Nakazaki, and A. Azuma, Phys. Rev. A 60, R17 (1999).

15. S.E. Canton-Rogan et al., Phys. Rev. Lett. 85, 3113 (2000).

16. T.W. Gorczyca and F. Robicheaux, Phys. Rev. A 60, 1216 (1999).

\section{Chien-Nan Liu}

Max Planck Institute for the Physics of Complex Systems

Nöthnitzer Str. 38

D-01187 Dresden, Germany

\section{Anthony F. Starace}

Department of Physics and Astronomy

The University of Nebraska

Lincoln, Nebraska 68588-0111 U.S.A.

e-mail: astaracel@unl.edu 